\title{
Sigelliste und Abkürzungen
}

\section{Sigelliste}

In der folgenden Übersicht sind alle Handschriften verzeichnet, die für die textkritische Arbeit herangezogen wurden. Bei den meisten dieser Handschriften handelt es sich um Katenenhandschriften, die auch für die Editionen der Fragmente der Psalmenkommentierung des Origenes und Eusebius relevant sind. Für diese Handschriften wurden genau die Siglen verwendet, unter denen sie auch in den zukünftigen Editionen unseres Vorhabens erscheinen werden. Bei der Vergabe dieser Siglen wurden vor allem die Verwandschaftsverhältnisse der Handschriften untereinander berücksichtigt. Denjenigen Handschriften, die nur für einzelne Prologtexte im vorliegenden Band, aber eben nicht für die weitere Psalmenkommentierung der beiden Kirchenväter von Interesse sind, wurden dagegen gar keine, oder aber Siglen zugewiesen, aus denen sich dieser Status leicht erkennen lässt, in der Regel eine Abkürzung der Handschriftenbezeichnung.

Neben der Datierung sind für alle Handschriften, auf die es zutrifft, die Typen nach der Klassifizierung durch Karo/Lietzmann ${ }^{1}$ angegeben. Die Angaben in Klammern betreffen Handschriften, die von Karo/Lietzmann nicht in ihren Catalogus aufgenommen wurden, sich aber eindeutig einem der dort verzeichneten Katenentypen zuordnen lassen. Es wurde darauf verzichtet, die Ergebnisse von Gilles Dorivals grundlegender Untersuchung der Psalmenkatenen aufzunehmen, da eine sachgerechte Darstellung den Rahmen der Liste sprengen würde. Zudem hat Dorival die Prologtexte aus seinen Studien bewusst ausgenommen. Dennoch ist anzumerken, dass seine Ergebnisse im Hinblick auf die Einordnung der Handschriften im Großen und Ganzen auch auf das Prologmaterial anwendbar sind. Mit dem Handschriftenindex am Ende des kürzlich erschienenen Bandes $V^{2}$ steht nun auch ein ausgezeichnetes Instrument für einen schnellen Zugang zu den sehr detaillierten Handschriftenbeschreibungen und Analysen des Inhalts der Katenen zur Verfügung.

1 Vgl. Karo/Lietzmann, Catalogus, 21-66.

2 Dorival, Chaînes V, 403-411. 


\begin{tabular}{|c|c|c|c|}
\hline Alex & London. British Library Royal MS 1 D VII & 5. Jh. & -3 \\
\hline Auct & Ox. Bodl. Auct. D. 4.1. & 951 & I \\
\hline Ba & Ox. Bodl. Barocc. gr. 235 & 9./10. Jh. & VI \\
\hline $\mathbf{B b}$ & Vat. gr. 1789 & 10./11. Jh. & VI \\
\hline Da & Paris. gr. 146 & 10. Jh. & $\mathrm{XV}$ \\
\hline Dc & Vat.gr. 1422 & 10. Jh. & XV \\
\hline De & Med. Ambros. C 98 sup & 12. Jh. & XVI \\
\hline Df & Flor. Laur. VI 3 & 11. Jh. & $(\mathrm{XVI})$ \\
\hline $\mathbf{E}$ & Paris. Coisl. 44 & 10. Jh. & -4 \\
\hline $\mathbf{F}$ & Vat. gr. 752 & 1090 & XXII \\
\hline Ja & Hieros. S. Cruc. 1 & 15. Jh. & $(\mathrm{X})$ \\
\hline Jb & Ox. Bodl. Barocc. gr. 223 & 15. Jh. & $\mathrm{X}$ \\
\hline Jc & Vat. Borg.gr. 2 & 1665 & (X) \\
\hline $\mathbf{K}$ & Vat. Ottob.gr. 398 & 11. Jh. & IV \\
\hline Lc & Paris. gr. 163 & 11. Jh. & XVII \\
\hline Ld & Vat.gr. 744 & 10. Jh. & XVII \\
\hline Le & Vat.gr. 1617 & 15. Jh. & XVIIa \\
\hline $\mathbf{M}$ & Mosq.gr. 358 & 11. Jh. & XVIII \\
\hline M47 & Med. Ambros. M 47 sup. & 12. Jh. & - \\
\hline $\mathbf{0}$ & Vindob. theol.gr. 59 & 13. Jh. & VII \\
\hline $\mathbf{R a}$ & Paris. gr. 169 & 14. Jh. & XXIV \\
\hline $\mathbf{R b}$ & Taur. B VII 30 (=B I 10) & 8. Jh. & XXVI \\
\hline Reg & Vat.Reg.gr. 1 & 10. Jh & -5 \\
\hline $\mathbf{S}$ & Paris. Coisl. 12 & 11./13. Jh. & XXVII \\
\hline Ta & Monac.gr. 252 & 10./11. Jh. & XVIIb \\
\hline $\mathbf{T b}$ & Taur. B I 22 & 1049 & XVIIb \\
\hline $\mathbf{U}$ & Mosq.gr. 194 & 10.-11. Jh. & XIII \\
\hline $\mathbf{V}$ & Vat.gr. 754 & 10. Jh. & XIIIv \\
\hline Wc & Athos Esphigm. 73 & 13. Jh. & $\mathrm{V}$ \\
\hline $\mathbf{X a}$ & Vat.gr. 341 & 1021 & - \\
\hline $\mathbf{X b}$ & Vat.gr. 1866 & 13. Jh. & - \\
\hline Xc & Paris. gr. 143 & 12. Jh. & XXV \\
\hline
\end{tabular}

3 Diese Handschrift enthält keine Katene, vielmehr handelt es sich um den Teil des Codex Alexandrinus, der die Psalmen enthält.

4 Dies ist keine Katene, sondern die einzige Handschrift der direkten Überlieferung des Eusebianischen Kommentars zu Ps 51-95.

5 Bei dieser Handschrift handelt es sich um eine alte Bibelhandschrift. 
xxiv — Sigelliste und Abkürzungen

$\begin{array}{llll}\text { Xd } & \text { Rom. Casanat. } 1908 & \text { 13. Jh. } & \text { XXV } \\ \text { Xe } & \text { Vat. Barb. gr. } 340 & 10 . \text { Jh. } & \text { XII } \\ \text { Xm } & \text { Vat. gr. } 342 & 1087 / 88 & - \\ \text { Za } & \text { Med. Ambros. B 106 sup. HP 113 } & 967 & \text { XIV } \\ \text { Zb } & \text { Vat. gr. } 1747 & 10 . / 11 . \text { Jh. } & \text { XIV }\end{array}$

\section{Abkürzungen}

Im vorliegenden Band werden (außer den allgemein üblichen) folgende Abkürzungen verwendet:

cett $=$ die übrigen Codices

codd $=$ alle Codices

tit. $=$ Überschrift

prol. $=$ Prologus

Z. $=$ Zeile

Desweiteren ist darauf hinzuweisen, dass Stellen im Alten Testament, insbesondere die Psalmen, sofern nichts anderes angegeben ist, nach der Septuaginta zitiert werden. Wenn nach der Hebräischen Bibel zitiert wird, ist das durch (M) gekennzeichnet; Zitaten aus der Hexapla ist jeweils der Anfangsbuchstabe der Version beigegeben : 'A für Aquila, $\Sigma$ für Symmachos und $\Theta$ für Theodotion. 